\title{
How much equipment is prescribed for people with spinal cord injury in Australia, do they use it and are they satisfied 1 year later?
}

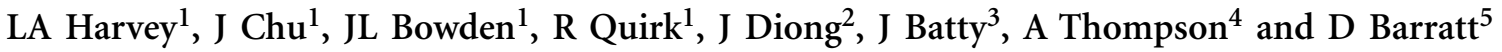

Background and purpose: The purpose of this study was to determine the amount and type of equipment prescribed to aid mobility and self-care for patients with spinal cord injuries (SCl) in Australia and to determine how frequently the equipment is used 1 year later and whether patients are satisfied with it.

Methods: A consecutive series of 61 patients admitted for rehabilitation to two Sydney $\mathrm{SCl}$ units were recruited. All mobility and self-care equipment worth more than $\$ A \cup 50$ prescribed by therapists before patients' discharge was recorded. This included wheelchairs, commodes, shower chairs, hoists, electric beds, pressure-relieving cushions, bed mattresses, slideboards, walking aids, orthoses, electrical stimulation systems and other pieces of notable exercise equipment. Patients were interviewed 1 year later and asked about each piece of equipment they had been prescribed. Specifically, they were asked about how frequently they had used each piece of equipment and whether they were satisfied with it. Data were analysed using descriptive statistics.

Results: Three hundred and fourteen pieces of equipment, including 68 wheelchairs, were prescribed for the 61 patients. Most of the equipment (226/314) was used more than 20 times in the 2 months preceding the 1-year review. Most patients were satisfied or highly satisfied with the majority of equipment prescribed, although patients were very dissatisfied or only partly dissatisfied with $18 / 314$ pieces of equipment.

Discussion: Patients were generally satisfied with the equipment they were prescribed. There was a small amount of equipment prescribed that was not used 1 year later or with which patients were dissatisfied.

Spinal Cord (2012) 50, 676-681; doi:10.1038/sc.2012.28; published online 27 March 2012

Keywords: equipment; wheelchair; rehabilitation

\section{INTRODUCTION}

Equipment prescription is an important aspect of successful and costefficient rehabilitation for people with spinal cord injuries (SCI). ${ }^{1,2}$ Typically, therapists prescribe equipment to aid mobility, self-care and independence. The extent and type of equipment prescribed is partly determined by funding. In one state of Australia, New South Wales, all mobility and self-care equipment worth more than \$AU100 is provided by the state government free of charge to all Australian citizens to facilitate discharge from hospital following recent SCI unless an individual has a very high annual income. Essential equipment includes walking aids, wheelchairs, adjustable beds, hoists, showers and toileting aids. Examples of equipment not provided by the government but sometimes self-funded or provided by private insurance companies include: small inexpensive items, recreational or sporting equipment, standing wheelchairs, electrical stimulation systems, cycles, parallel bars and tilt tables.

There is little communication between policy makers within and outside Australia about appropriate equipment prescription following SCI. ${ }^{3-5}$ For example, in some parts of Australia and in other similar western countries, a second manual wheelchair is considered essential for a person primarily reliant on a power wheelchair to enable access to private vehicles. Similarly, sometimes electric beds and hoists are considered essential when at other times they are not. Clearly, policies about equipment prescription are primarily driven by the socioeconomic status of a country and the priority a country places on social justice. ${ }^{6}$

We were interested in quantifying the amount and range of mobility and self-care equipment typically prescribed to people with SCI from one region of Australia. This information will help pave the way for comparisons within and outside Australia and will help drive the development of international consensus and guidelines on appropriate equipment prescription for people with SCI. The appropriateness of equipment can be partly gauged by determining how frequently the equipment is used and how satisfied patients are with the equipment 1 year later. ${ }^{7}$ The purpose therefore of this study was to determine the amount and range of mobility and self-care equipment prescribed for people with SCI soon after injury and to determine how frequently the equipment was used 1 year later and whether patients were satisfied with the equipment.

${ }^{1}$ Rehabilitation Studies Unit, Sydney Medical School Northern, University of Sydney, Sydney, New South Wales, Australia; ${ }^{2}$ The George Institute for Global Health, Sydney, New South Wales, Australia; ${ }^{3}$ Spinal Unit, Prince of Wales Hospital, Sydney, New South Wales, Australia; ${ }^{4}$ Moorong Spinal Unit, Royal Rehabilitation Centre Sydney, Ryde, New South Wales, Australia and ${ }^{5}$ Royal North Shore Hospital, Sydney, New South Wales, Australia

Correspondence: A/Professor LA Harvey, Rehabilitation Studies Unit, Royal Rehabilitation Centre Sydney, PO Box 6, Ryde, New South Wales 1680, Australia.

E-mail: lisa.harvey@sydney.edu.au.

Received 23 December 2011; revised 2 February 2012; accepted 17 February 2012; published online 27 March 2012 


\section{METHODS}

All patients admitted to two Sydney SCI units between March 2009 and March 2010 were screened for inclusion. Patients were eligible for inclusion if they had sustained a recent SCI and were undergoing an initial bout of rehabilitation. They were excluded if they had minimal disability and were discharged with less than \$AU50 worth of equipment. Patients on mechanical ventilation who were yet to be discharged from hospital 1 year after injury were also excluded. The study received ethical approval from the appropriate institutions and informed consent was obtained from all patients. The institutional and governmental regulations concerning the ethical use of human volunteers were followed during the course of this research.

Government regulations mandate that all patients with suspected SCI from the state of New South Wales (with a population of 6 million) are managed at one of the two SCI units included in this study. Hence, these two SCI units reflect nearly all people with recent SCI from the state of New South Wales. The two SCI units are government funded where patients are treated equally and at no cost to the individual. All equipment essential for mobility and selfcare are provided by either government or private funding schemes. The only people who do not receive mobility and self-care equipment through one of these schemes are the very wealthy (less than $5 \%$ of patients included in this cohort) or non-Australian citizens.

All equipment worth more than \$AU50 that was commonly prescribed by therapists for mobility or self-care before discharge from hospital was recorded. This included wheelchairs, commodes, shower chairs, hoists, electric beds, pressure-relieving cushions, bed mattresses, slideboards, walking aids, orthoses, electrical stimulation systems and other pieces of notable exercise equipment. It did not include computers, cars and adaptations or modifications to cars and homes, which are also sometimes funded. Sporting and recreational equipment, including sports wheelchairs, are not typically funded or prescribed by therapists' before discharge and were not included. The source of funding and cost of each piece of equipment was also recorded.

Patients were interviewed 1 year after injury. Patients were asked to separately rate each piece of equipment according to how much they had used it and how satisfied they were with it. Usage was estimated by patients using the following 4-point scale designed for the purpose of this study:

(1) Not used in the last 2 months

(2) Used less than five times in the last 2 months (that is, less than once per fortnight)

(3) Used between five and twenty times in the last 2 months (that is, one to five times per fortnight)

(4) Used twenty or more times in the last 2 months (that is, more than five times per fortnight)

Satisfaction was recorded on the following 5-point scale, again specifically designed for the purpose of this study:

(1) Not at all satisfied

(2) Not very satisfied

(3) More or less satisfied

(4) Quite satisfied

(5) Very satisfied

If patients expressed any dissatisfaction with a piece of equipment, they were asked open-ended questions exploring reasons for dissatisfaction.

\section{RESULTS}

One hundred and twenty-two patients were admitted to the two SCI units and screened for inclusion over the 1-year study period but only 61 patients met the inclusion criteria and/or were followed up 1 year later. The reasons for exclusion or failure to follow up at 1 year were: unable to be contacted or overseas at 1 year $(n=11)$, declined to be involved $(n=6)$, deceased 1 year after injury $(n=9)$, still in hospital
1 year after injury $(n=12)$, under 18 years of age $(n=2)$, self discharged before equipment was prescribed $(n=1)$, minimal disability that did not require equipment $(n=11)$, or discharged to a non-spinal unit or hospital outside of the state before equipment was prescribed $(n=9)$. Fifty two patients were male and 9 were female (see Table 1). The median (interquartile range) age at the time of injury was 40 years (25-61). Thirty four patients had motor complete lesions (that is, AIS A or AIS B) and 36/61 had a neurological level of T1 or higher. All but six patients had motor scores of 75/100 or less, and 40 patients had motor scores of $50 / 100$ or less. Fifty patients $(82 \%)$ had traumatic injuries and 11 patients (18\%) had nontraumatic spinal cord lesions.

Three hundred and fourteen pieces of equipment were prescribed for the 61 patients, including 42 manual wheelchairs, 26 power wheelchairs, 17 hoists, 19 electric beds and 30 bed mattresses (see Table 2). Other equipment prescribed included walking aids and orthoses, pressure-relieving cushions, exercise equipment and miscellaneous items. Sixteen patients were prescribed two wheelchairs each (see Table 3). Most of these patients were primarily reliant on hand control power wheelchairs and had a second manual wheelchair as backup. Ten patients had extensive paralysis (that is, AIS A, B or C, T1 and above) and three patients were over 65 years of age.

\section{Table 1 Characteristics of patients}

\begin{tabular}{lc} 
Age (years) & $40(25-61)$ \\
\hline Gender (M:F) & $52: 9$
\end{tabular}

Association Impairment Scale (AIS), $\mathrm{n}$

AIS A

AIS B

AIS C

AIS D

Neurological level, $\mathrm{n}$

C5 and above

C6 to $\mathrm{T} 1$

32

T2 to T12

$\mathrm{L} 1$ and below

Motor score/100, n (\%)

$<5$

$5-25$

15

25-50

50-75

$>75$

Sources of funding for equipment, $\mathrm{n}(\%)^{\mathrm{a}}$

Life Time Care and Support Scheme (government) 11

EnableNSW Scheme (government)

Workers' compensation scheme (government or commercial)

Other insurance scheme (commercial)

Self-funded

Other

Type of injury $\mathrm{n}(\%)$

Traumatic

Non-traumatic

18

All data are reported as median (interquartile range) unless otherwise stated.

a Unable to determine primary funding source for one patient. 
The source of equipment funding for patients is provided in Table 1. Forty eight had essential equipment funded through some type of government funding scheme (that is, Enable NSW, The NSW Lifetime Care and Support Scheme or The Workers Compensation Scheme) and four patients had essential equipment funded by a non-government insurance scheme. Some patients self-funded or sought funding from other sources (for example, donations) for additional non-essential equipment not provided by their primary funder. Seven patients self-funded or attained funding from other sources for essential and non-essential equipment either because the total cost of the equipment was small, they were not an Australian citizen or because they had a high annual income.

The median (interquartile range) cost of each piece of equipment is shown in Table 2. The most expensive pieces of equipment were the chin-control power wheelchairs (median, \$AU 25606) and the handcontrol power wheelchairs (median, \$AU 14917). There was a large variation in the cost of manual wheelchairs (interquartile range, \$AU 3370-\$AU 7198) and ankle-foot orthoses (interquartile range, \$AU 82-\$AU 803).

Most equipment $(n=226 / 314)$ was used more than 20 times in the 2 months preceding the 1 year assessments (that is, more than five times per fortnight). The exceptions were $8 / 26$ slideboards, $2 / 3$ electrical stimulation units and 4/14 shower chairs that were never used in two months preceding patients' 1-year assessments (see Table 2). Of 42 manual wheelchairs, 15 were used 20 or less times in the 2 months preceding patients' 1-year assessments (that is, less than five times per fortnight). However, 11 belonged to patients who primarily relied upon hand-control power wheelchairs and used a second manual wheelchair for backup (see Table 4).

Patients were 'satisfied' or 'very satisfied' with 247/314 (79\%) pieces of prescribed equipment. Patients were only dissatisfied with 18/314 (6\%) pieces of equipment (see Table 2). Reasons for dissatisfaction were categorised in the following ways:

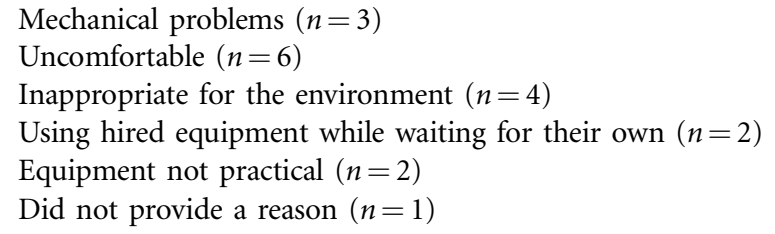

The most important and common pieces of equipment patients were dissatisfied with were manual wheelchairs (4/42) and shower chairs (3/14). The main criticism with equipment was that it was unsuitable for the environment $(n=4)$ or uncomfortable $(n=6)$.

\section{DISCUSSION}

The results of this study show for the first time the type and amount of equipment prescribed to a representative cohort of people with SCI in Australia. The information is important because it provides a benchmark for comparisons with other countries ${ }^{8}$ and helps pave the way for discussions about policies on appropriate equipment prescription. The results also provide an indication of the typical cost of equipment. Cost is an important consideration that needs to be included in any analysis of the costs involved in initially setting up an individual with SCI in the community. A comparable study examined costs of all 'durable medical equipment' for privately insured patients in the United States in the first year since injury, but costs were not itemised and included modifications to cars and homes. ${ }^{9}$
Patients were 'quite' or 'very satisfied' with $247 / 314$ (79\%) pieces of prescribed equipment, and only dissatisfied with 18/314 (6\%) pieces of prescribed equipment. The most important and common pieces of equipment patients were dissatisfied with were manual wheelchairs $(4 / 42)$ and shower chairs (3/14), although the rate of dissatisfaction with wheelchairs was much lower than in a comparable Dutch study. ${ }^{1}$ The main criticism with equipment was that it was unsuitable for the environment $(n=4)$ or uncomfortable $(n=6)$. Of course, it is possible that dissatisfaction with wheelchairs and other pieces of equipment may have been higher if patients were assessed 5-10 years after injury when equipment had started to fail and when funding for replacement equipment is more difficult to attain. In addition, we did not capture equipment that might have been prescribed to aid communication (for example, computers) or transportation (for example, modifications to cars). Nor did we look at equipment prescribed to patients on ventilators who were still in hospital 1 year after injury. Instead, we focused on equipment prescribed for mobility and self-care and to those discharged within a year of injury.

Admittedly, our assessment of satisfaction with equipment was crude. We only examined satisfaction at 1 year after injury and do not know about satisfaction before or after this point. In addition, we only asked patients to rate satisfaction on a 5-point scale and did not ask them to distinguish between their satisfaction with different aspects of the equipment. For example, some patients may have been highly satisfied with the aesthetics of a piece of equipment but highly dissatisfied with its comfort. We only sought one overall measure of satisfaction because we asked patients about their satisfaction with every piece of equipment they had been prescribed. Some patients had 10 or more pieces of equipment and we did not want to exhaust their goodwill by asking extensive questions about each piece of equipment. Our decision to use one question to gauge satisfaction was based on the assumption that when patients are asked one question, they intuitively think about the feature of a piece of equipment that is most important to them. In this way, the one overall rating provides a way of weighting responses according to what is important to the patient. For example, if comfort is of utmost importance, as suggested by some, ${ }^{7,10,11}$ then this would probably be the main thing a patient would think about when rating satisfaction, and vice versa if aesthetics is most important. Our justification for this approach comes from the widespread use of a similar concept when gauging patients' impressions of change in response to a medical intervention. Rather than asking patients to rate every aspect and implication of an intervention, patients are asked to provide a global impression of change. ${ }^{12}$ There are however very comprehensive questionnaires that capture all aspects of users' satisfaction with equipment. ${ }^{13}$ The most commonly used are the Quebec User Evaluation of Satisfaction with Assistive Technology $7,14,15$ and the Psychosocial Impact of Assistive Devices. ${ }^{6,16}$ Both of these assessments are more conducive to studies designed to determine the usefulness of a particular piece of equipment. For example, a number of studies have used these assessments to gauge user satisfaction with wheelchairs. ${ }^{11,17,18}$ These assessments were not used in this study because they are very extensive and time consuming to administer and not appropriate if trying to get a broad impression of user satisfaction across a wide range of equipment as done in this study.

Satisfaction with equipment was also gauged by examining usage. Generally, equipment was well used. However, usage data were based on self-report so patients may have over or under-estimated how frequently the equipment was used. In addition, usage data only captured equipment used in the 2 months preceding the 1 -year review 


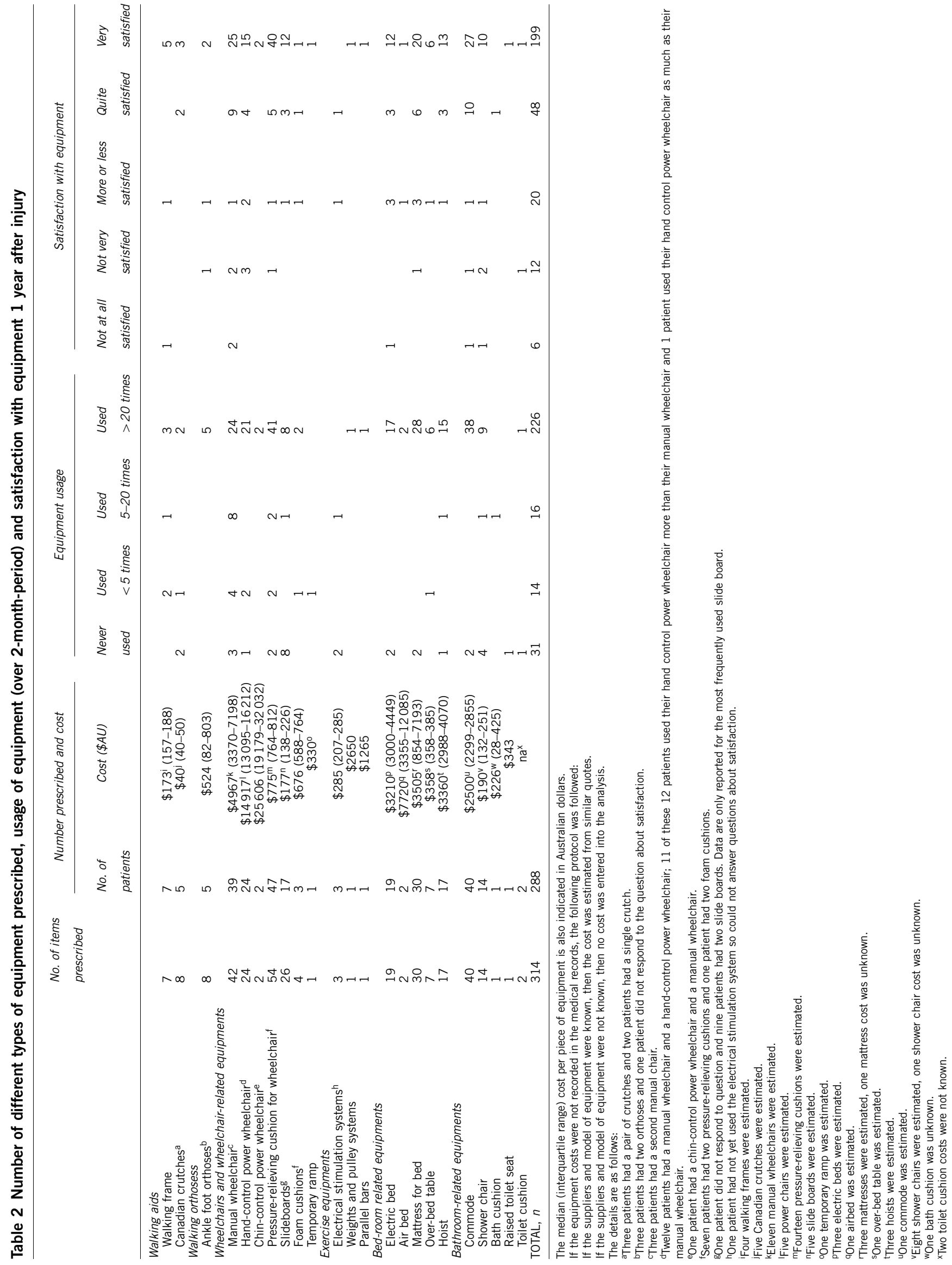


Table 3 Neurological details of patients provided with two wheelchairs $(n=16)$

\begin{tabular}{llllll}
\hline Primary & $\begin{array}{l}\text { Secondary } \\
\text { wheelchair }\end{array}$ & Age & AlS & $\begin{array}{c}\text { Neurological } \\
\text { level }\end{array}$ & $\begin{array}{c}\text { Motor } \\
\text { score }\end{array}$ \\
\hline Hand control power & Manual wheelchair & 76 & A & T3 & 50 \\
Hand control power & Manual wheelchair & 65 & D & C5 & 42 \\
Hand control power and manual wheelchair & 50 & B & C5 & 13 \\
Chin control power and manual wheelchair & 35 & A & C5 & 43 \\
Hand control power & Manual wheelchair & 19 & A & C4 & 4 \\
Hand control power & Manual wheelchair & 26 & B & C5 & 26 \\
Hand control power & Manual wheelchair & 71 & C & T10 & 53 \\
Hand control power & Manual wheelchair & 23 & A & C4 & 6 \\
Hand control power & Manual wheelchair & 25 & B & C4 & 6 \\
Hand control power & Manual wheelchair & 27 & B & C4 & 7 \\
Hand control power & Manual wheelchair & 44 & D & C3 & 55 \\
Hand control power & Manual wheelchair & 49 & C & C4 & 16 \\
Hand control power & Manual wheelchair & 34 & B & C5 & 13 \\
Manual wheelchair & Manual wheelchair & 18 & A & T1 & 50 \\
Manual wheelchair & Manual wheelchair & 26 & A & T6 & 50 \\
Manual wheelchair & Manual wheelchair & 26 & C & L1 & 52 \\
\hline
\end{tabular}

The patient was unable to identify one chair as primary and the other as secondary.

Table 4 The usage and satisfaction with back-up manual wheelchair for the 11 patients who primarily used a power hand control wheelchair

\begin{tabular}{lcccccccc}
\hline Usage & \multicolumn{1}{c}{ Satisfaction } \\
\hline & Used & Used & Used & Not & Not & More \\
Never & $<5$ & $5-20$ & $>20$ & at all & very & or less & Quite & Very \\
used & times & times & times & satisfied satisfied satisfied satisfied satisfied \\
\hline 2 & 3 & 6 & & 1 & 1 & 4 & 5 \\
\hline
\end{tabular}

and did not capture all aspects of the usefulness of equipment. A piece of equipment may be used very infrequently but may have major implications on independence and quality of life. For example, 15/42 manual wheelchairs were used 20 or less times in the 2 months preceding the 1-year reviews (that is, less than five times per fortnight). However, 11 belonged to patients who primarily relied upon hand-control power wheelchairs and only occasionally used a manual wheelchair (see Table 4). Hence, although these wheelchairs were not often used, they may have provided patients with greater flexibility and choice. For instance, the manual wheelchairs may have enabled patients to access private vehicles increasing their transport options. Similarly, the prescription of two power wheelchairs may have ensured that when the primary power wheelchair malfunctioned and required repairs, patients had access to an alternative. ${ }^{19}$ Some countries such as Denmark and Netherlands have in the past typically provided patients with two wheelchairs, although it is not clear whether this is still the case or how many other countries do the same. ${ }^{20}$ Policy decisions about second wheelchairs and other optional pieces of equipment should be underpinned by countryspecific economic evaluations. These can be done in one of four ways, namely through cost-minimisation analyses, cost-effectiveness analyses, cost-utility analyses or cost-benefit analyses. ${ }^{6,21}$ For example, in one case study done back in 1993, authors estimated that a power wheelchair for an individual would cost 1417 euro per year more than a manual wheelchair and would result in a gain of 0.012 qualityadjusted life years. ${ }^{3}$ Little work has been done since this time to evaluate the cost benefit of difference pieces of equipment, although this type of research is important for policy makers. ${ }^{5}$

The high rate of equipment usage and satisfaction with equipment may have been in part due to the systems of rehabilitation in Australia. For example, patients generally receive between 2 and 5 months of inpatient rehabilitation at which time most equipment is prescribed. This is a longer length of inpatient hospital stay than provided in some countries. ${ }^{22}$ One benefit of longer inpatient hospital stay is that it provides sufficient time and opportunity for careful consideration of equipment needs. In addition, in Australia, equipment is only prescribed by the treating physiotherapists and occupational therapists responsible for daily therapy. It is not prescribed, like in some countries, by independent therapists nominated by the funders, nor is it prescribed on an outpatient basis. Our systems help ensure that therapists are well acquainted with the patients' needs and priorities. Another important aspect of equipment prescription in Australia is therapists' freedom to prescribe any type or make of equipment from any supplier provided the cost is reasonable and can be justified according to local guidelines. ${ }^{4}$ This policy gives therapists prescribing equipment considerable autonomy perhaps similar to that noted in countries such as Sweden ${ }^{11}$ and Denmark who also report high rates of satisfaction and equipment usage. ${ }^{8}$ It also enables and encourages patients to trial different types of equipment empowering them and possibly contributing to satisfaction. ${ }^{2}$ Needless to say trialling different types of equipment is a time consuming process and cannot be easily done on an outpatient basis. Nor can it be done effectively if patient length of hospital stay is short. Perhaps future studies could explore some of these issues. Studies which compare patients from different countries and their satisfaction with equipment may be particularly useful.

The results of this study only scrape the surface of an important area of research that has received little attention to date. Our sample was small precluding statistical comparisons of subgroups but it was representative of an Australian population. The results raise broad questions about determinants of patient satisfaction with equipment, the influence of government policies and procedures on equipment selection, and the importance of appropriate equipment prescription for quality of life. There is a need for increased global collaboration on equipment prescription, policies and standards.

\section{DATA ARCHIVING}

There were no data to deposit.

\section{CONFLICT OF INTEREST}

A/Professor Lisa Harvey works at an academic institute, which is primarily funded by the NSW Lifetime Care and Support Scheme. The NSW Lifetime Care and Support Scheme is a government-funded organisation, which funds equipment, lifetime care and rehabilitation for all people seriously injured in motor vehicle accidents regardless of fault, including people with SCI. The remaining authors declare no conflict of interest.

\section{ACKNOWLEDGEMENTS}

Thanks to the patients and therapists from the two Sydney SCI Units who participated. This study was funded by the Rehabilitation and Disability Research Foundation and the NSW Lifetime Care and Support Scheme. 
1 Post MW, van Asbeck FW, van Dijk AJ, Schrijvers AJ. Services for spinal cord injured: availability and satisfaction. Spinal Cord 1997; 35: 109-115.

2 Di Marco A, Russell M, Masters M. Standards for wheelchair prescription. Aust Occup Ther J 2003; 50: 30-39.

3 Brodin J, Persson J. Cost-utility analysis of assistive technologies in the European Commission's TIDE Program. Technology Initiative for Disabled and Elderly people. Int J Technol Assess Health Care 1995; 11: 276-283.

4 Enable NSW and Lifetime Care and Support Authority. Guidelines for the Prescription of a Seated Wheelchair or Mobility Scooter for People with Traumatic Brain Injury or Spinal Cord Injury, 2011: Sydney.

5 Layton N, Wilson E, Colgan S, Moodie M, Carter R. The Equipping Inclusion Studies: Assistive Technology Use and Outcomes in Victoria 2010: Deakin University, Burwood.

6 Jacobs P, Hailey D, Jones A. Economic evaluation for assistive technology policy decisions. J Disab Policy Stud 2003; 14: 119-125.

7 Samuelsson K, Wressle E. User satisfaction with mobility assistive devices: an important element in the rehabilitation process. Disabil Rehabil 2008; 30: 551-558.

8 Biering-Sørensen T, Hansen RB, Biering-Sørensen F. Home aids and personal assistance 10-45 years after spinal cord injury. Spinal Cord 2009; 47: 405-412.

9 Young AE, Webster B, Giunti G, Pransky G, Nesathurai S. Services provided following compensable work-related tetraplegia. Spinal Cord 2004; 42: 248-260.

10 Weiss-Lambrou R, Tremblay C, LeBlanc R, Lacoste M, Dansereau J. Wheelchair seating aids: how satisfied are consumers? Assist Technol 1999; 11: 43-53.

11 Bergström AL, Samuelsson K. Evaluation of manual wheelchairs by individuals with spinal cord injuries. Dis Rehabil: Ass Tech 2006; 1: 175-182.

12 Studenski S, Hayes RP, Leibowitz RQ, Bode R, Lavery L, Walston J et al. Clinical global impression of change in physical frailty: development of a measure based on clinical judgment. J Am Geriatr Soc 2004; 52: 1560-1566.
13 Wressle E, Samuelsson K. User satisfaction with mobility assistive devices. Scand J Occup Ther 2004; 11: 143-150.

14 Demers L, Weiss-Lambrou R, Ska B. Development of the Quebec User Evaluation of Satisfaction with assistive Technology (QUEST). Assist Technol 1996; 8: 3-13.

15 Wessels RD, de Witte LP, van den Heuvel WJA. Measuring effectiveness of and satisfaction with assistive devices from a user perspective: an exploration of the literature. Technol Disabil 2004; 16: 83-90.

16 Day H, Jutai J. Measuring the psychosocial impact of assistive devices: the PIADS. Can J Rehabil 1996; 9: 159-168.

17 Chan SC, Chan AP. User satisfaction, community participation and quality of life among Chinese wheelchair users with spinal cord injury: a preliminary study. Occup Ther Int 2007; 14: 123-143.

18 De Groot S, Post MWM, Bongers-Janssen HMH, Bloemen-Vrencken JH, Van Der Woude LHV. Is manual wheelchair satisfaction related to active lifestyle and participation in people with a spinal cord injury? Spinal Cord 2011; 49: 560-565.

19 Mcclure LA, Boninger ML, Oyster ML, Williams S, Houlihan B, Lieberman JA et al. Wheelchair repairs, breakdown, and adverse consequences for people with traumatic spinal cord injury. Arch Phys Med Rehabil 2009; 90: 2034-2038.

20 Biering-Sørensen F, Hansen RB, Biering-Sørensen J. Mobility aids and transport possibilities 10-45 years after spinal cord injury. Spinal Cord 2004; 42 : 699-706.

21 Fuhrer MJ. Assessing the efficacy, effectiveness, and cost-effectiveness of assistive technology interventions for enhancing mobility. Dis Rehabil: Ass Tech 2007; 2 : 149-158.

22 Taylor-Schroeder S, LaBarbera J, McDowell S, Zanca JM, Natales A, Mumma S et al. Physical therapy treatment time during inpatient spinal cord injury rehabilitation. J Spinal Cord Med 2011; 34: 149-161. 